\title{
Avaliação clínica e tomográfica em crianças portadoras de Leishmaniose Visceral Humana
}

\author{
Clinical and tomographic evaluation in children with Human Visceral Leishmaniosis
}

Evaluación clínica y tomográfica en niños con Leishmaniosis Visceral Humana

Camila Mendonça França ${ }^{1 *}$, Ana Jovina Barreto Bispo', Larissa Goes Azevedo¹, Lucas Thierry Costa Silva ${ }^{1}$, Maria Luiza Doria Almeida'.

\section{RESUMO}

Objetivo: Avaliar a existência de sintomatologia respiratória em pacientes pediátricos com Leishmaniose Visceral (LV), identificar alterações tomográficas e associar sintomas respiratórios e acomentimento pulmonar. Métodos: Trata-se de um estudo observacional, com abordagem quantitativa e delineamento transversal não comparado avaliando 22 pacientes com diagnóstico confirmado de LV internados na enfermaria pediátrica de um hospital universitário, entre janeiro de 2015 e julho de 2018. Os usuários foram investigados quanto à sintomatologia por meio de anamnese e de exame físico e, posteriormente foram encaminhados para realização de radiografia e Tomografia Computadorizada de Alta Resolução (TCAR) de tórax. Resultados: A média de idade foi de 6,9 anos e predominou o gênero masculino (59\%). A frequência de manifestações respiratórias foi de $27,3 \%$. O sintoma respiratório mais observado foi a tosse $(22,7 \%)$, sendo em $18,2 \%$ dos casos produtiva e em $4,5 \%$ seca. O segundo sintoma respiratório foi a taquipneia $(9,1 \%)$. Não foram evidenciados dor torácica ou outros sintomas respiratórios. A TCAR foi considerada alterada em $50 \%$ dos casos. Não foi encontrada associação entre os sintomas respiratórios e alterações tomográficas. Conclusão: Conclui-se que sintomas respiratórios são frequentes em crianças com LV, assim como o comprometimento pulmonar demonstrado através das alterações tomográficas.

Palavras-chave: Leishmaniose visceral, Sinais e sintomas respiratórios, Crianças, Lesão pulmonar.

\begin{abstract}
Objective: To evaluate the existence of respiratory symptoms in pediatric patients with Visceral Leishmaniasis (VL), to identify tomographic changes and to associate respiratory symptoms and lung involvement. Methods: This is an observational study, with a quantitative approach and cross-sectional design not compared evaluating 22 patients with confirmed diagnosis of $\mathrm{VL}$ admitted to the pediatric ward of a university hospital, between January 2015 and July 2018. Users were investigated for symptoms through anamnesis and physical examination and were subsequently referred for X-ray and high-resolution computed tomography (HRCT) of the chest. Results: The mean age was 6.9 years and the male gender predominated (59\%). The frequency of respiratory manifestations was $27.3 \%$. The most common respiratory symptom was cough $(22.7 \%), 18.2 \%$ of the cases being productive and $4.5 \%$ being dry. The second respiratory symptom was tachypnea $(9.1 \%)$. There was no evidence of chest pain or other respiratory symptoms. HRCT was considered altered in $50 \%$ of cases. No association was found between respiratory symptoms and tomographic changes. Conclusion: It is concluded that respiratory symptoms are frequent in children with $\mathrm{VL}$, as well as pulmonary impairment demonstrated through tomographic changes.
\end{abstract}

Key words: Leishmaniasis visceral, Signs and symptoms, respiratory, Children, Lung injury.

\section{RESUMEN}

Objetivo: Evaluar la existencia de síntomas respiratorios en pacientes pediátricos con Leishmaniasis Visceral (LV), identificar cambios tomográficos y asociar síntomas respiratorios y afectación pulmonar. Métodos: Se trata de un estudio observacional, con abordaje cuantitativo y diseño transversal no comparado evaluando 22 pacientes con diagnóstico confirmado de LV ingresados en la sala de pediatría de un hospital universitario, entre enero de 2015 y julio de 2018. Los usuarios fueron investigados por síntomas mediante anamnesis y

${ }^{1}$ Universidade Federal de Sergipe (UFS), Aracaju - SE. *E-mail: camilaamff@gmail.com

SUBMETIDO EM: 5/2021

ACEITO EM: 5/2021

PUBLICADO EM: 5/2021 
exploración física y posteriormente remitidos para radiografía y tomografía computarizada de alta resolución (TCAR) de tórax. Resultados: La edad media fue de 6,9 años y predominó el sexo masculino (59\%). La frecuencia de manifestaciones respiratorias fue del 27,3\%. El síntoma respiratorio más común fue la tos (22,7\%), en el $18,2 \%$ de los casos fue productivo y en el $4,5 \%$ seco. El segundo síntoma respiratorio fue la taquipnea $(9,1 \%)$. No hubo evidencia de dolor en el pecho u otros síntomas respiratorios. La TCAR se consideró alterada en el $50 \%$ de los casos. No se encontró asociación entre síntomas respiratorios y cambios tomográficos. Conclusión: Se concluye que los síntomas respiratorios son frecuentes en los niños con LV, así como la afectación pulmonar demostrada a través de cambios tomográficos.

Palabras clave: Leishmaniasis visceral, Signos y síntomas respiratorios, Niños, Lesión pulmonar.

\section{INTRODUÇÃO}

A leishmanione visceral humana é uma doença crônica e sitêmica causada por protozoários tripanosomatídeos do gênero Leishmania. Nas Américas, a Leishmania (Leishmania) chagasi é a espécie comumente envolvida na transmissão da Leishmaniose Visceral (LV), e, o cão (Canis familiaris) é seu principal reservatório em áreas urbanas. Sua transmissão ocorre pela picada dos vetores, os flebotomíneos, infectados pela Leishmania (L) chagasi. Não há transmissão de pessoa a pessoa (BRASIL, 2017).

É uma doença endêmica, tendo sido registrados surtos frequentes. Entre 2010 e 2018 foram registrados 33605 casos, sendo 14310 casos (42,5\%) na faixa etária de 0 a 9 anos (BRASIL, 2019). A maior incidência em crianças pode ser explicada por umar relativa imaturidade imunológica celular característica dessa fase, a qual pode ser agravada por desnutrição e uma maior exposição ao vetor no peridomicílio, tão comum nas áreas endêmicas (BRASIL, 2017).

Sua apresentação clínica varia de formas discretas e oligossintomáticas, a moderadas e graves que podem evoluir para o óbito em $90 \%$ dos casos, se não tratada (BRASIL, 2017). A forma oligossintomática manifesta-se com um quadro clínico leve, de pequena duração, aproximadamente 15 dias, podendo persistir por alguns meses, que normalmente evolui para a cura clínica espontânea. O quadro clássico da parasitose é caracterizado por febre de longa duração, perda de peso, astenia, adinamia, hepatoesplenomegalia, tosse seca, além de anemia, leucopenia e hipergamaglobulinemia (BURZA S, et al., 2018). Com a progressão da doença, podem surgir diarreia, icterícia, vômito e edema periférico (DE OLIVEIRA JM, et al., 2010).

O envolvimento pulmonar na LV ainda é pouco descrito na literatura. A tosse é um sintoma predominante e muito citado, tanto nas formas oligossintomáticas como na forma clássica, geralmente é seca e persistente, permanecendo inalterada e desaparecendo apenas após fim do tratamento (CASTRO BC e SILVA JM, 2009). A alteração histológica pulmonar mais descrita é a pneumonite intersticial. Experimentos realizados em hamsters infectados com $L$. donovani permitiram caracterizar o quadro de pneumonite com presença de edema, infiltrado inflamatório, espessamento focal múltiplo dos septos e fibrose septal focal (DUARTE MIS E CORBETT CEP, 1984). Quadro de pneumonite intersticial crônica, produtiva, difusa e intensa também foi confirmado em cães com LV através de infiltrado inflamatório composto principalmente por macrófaos, linfócitos e plasmócitos, além de fibroblastos e fibras colágenas, causando espessamento pleural (DUARTE $\mathrm{MI}$, et al., 1986). Este processo inflamatório intersticial poderia explicar a tosse persistente e também ser um fator determinante para crescimento bacteriano e infecção (DE OLIVEIRA JM ,et al., 2010).

As manifestações clínicas da LV infantil são semelhantes às presentes nos adultos. Nessa faixa etária, a doença pode frequentemente complicar-se com doenças bacterianas secundárias ou superinfecções, como pneumonia, sepse, otite média e infecções do trato urinário, podendo levar à morte. Sendo comum também infestações parasitárias intestinais e icterícia em pacientes pediátricos com LV (SINGH S, et al., 2017). Sintomas respiratórios no internamento já foram relacionados com pior prognóstico em crianças, por provável infecção pulmonar coexistente (DE QUEIROZ SAMPAIO MJA, et al., 2010).

Apesar da LV no Brasil acometer principalmente a população pediátrica e de sabermos que é frequente a presença de sintomas respiratórios em crianças, ainda não foram encontrados estudos que evidenciem o comprometimento pulmonar direto em crianças com LV. Dessa maneira, o presente estudo objetivou avaliar a existência de sintomatologia respiratória em pacientes pediátricos com LV, identificar alterações 
tomográficas e associar sintomas respiratórios e acomentimento pulmonar. O melhor conhecimento da doença, auxiliará na prevenção de complicações respiratórias e na redução da mortalidade nessa faixa etária.

\section{MÉTODOS}

Foi realizado um estudo do tipo observacional, analítico com delineamento transversal não comparado envolvendo pacientes pediátricos com diagnóstico confirmado de LV internados na enfermaria pediátrica de um hospital universitário da região Nordeste do Brasil, no período de janeiro de 2015 a julho de 2018. O hospital é referência estadual para o tratamento da LV. Considerou-se diagnóstico confirmado o encontro do parasito em aspirado de medula óssea, a positividade do teste rápido imunocromatográfico ou ainda reação de imunofluorescência reativa com título de 1:80 ou superior. Foram excluídos os pacientes transferidos de outras unidades de saúde e os que já tinham iniciado o tratamento específico para a parasitose no momento da abordagem.

Os responsáveis legais foram abordados na enfermaria, receberam explicação a respeito da pesquisa e de seus métodos e, após a concordância em participar, assinaram o Termo de Consentimento Livre e Esclarecido (TCLE), no qual consta: o objetivo do estudo; os procedimentos de avaliação; os riscos e benefícios; o caráter de voluntariedade da participação; e a responsabilidade por parte do avaliador, tendo sido respeitada a privacidade e a total confiabilidade dos dados. Foi realizada, em todos os pacientes, avaliação clínico-epidemiológica através de uma ficha de avaliação individual elaborada pela investigadora do estudo, contendo identificação, história familiar, história mórbida pregressa, história da doença atual e uso de medicamentos. Em seguida foi realizado o exame físico e a avaliação antropométrica, posteriormente os pacientes foram encaminhados para realização de Tomografia Computadorizada de Alta Resolução (TCAR) e radiologia de tórax. $O$ dados referentes ao tratamento, tempo de internação e desfecho final foram coletados nos prontuários dos pacientes após a alta.

A tomografia foi realizada de acordo com a disponibilidade do serviço de radiologia antes ou, no máximo, em até 48 horas do início do tratamento. Todos os exames foram feitos sem contraste em um tomógrafo 64 canais Toshiba aquillion, varrendo todo o tórax no sentido caudocranial em inspiração completa. Foram feitos cortes com espessura de $1,0 \mathrm{~mm}$, intervalo de tempo de $1,5 \mathrm{~s}$. Para reconstrução da imagem utilizou-se algoritmo de alta resolução com matriz de $512 \times 512$ pontos. Os laudos foram emitidos por radiologistas do hospital e posteriormente revisados por um outro radiologista, também pertencente ao quadro funcional do hospital. Em laudos discordantes foi considerado o diagnóstico do radiologista revisor.

A radiografia de tórax foi realizada nas incidências póstero-anterior e perfil, em inspiração máxima e com o tórax na posição vertical, nas primeiras 72 horas após a admissão. Os laudos foram emitidos por radiologistas do serviço.

Realizou-se uma análise exploratória dos dados. As variáveis categóricas foram descritas por meio de frequência absoluta e relativa. As variáveis quantitativas serão descritas por média, mínimo e máximo. As variáveis qualitativas foram resumidas em tabelas de frequência/contingência como valores (n) e porcentagens (\%) com respectivo Intervalo de Confiança (IC) para 95\%. Foi aplicado Teste Exato de Fisher para testar a associação entre variavéis.

Esse estudo foi realizado segundo os preceitos da Declaração de Helsinki e do Código de Nuremberg, respeitadas as Normas de Pesquisa Envolvendo Seres Humanos (Res. CNS 466/2012). O projeto foi autorizado pela diretoria de ensino e pesquisa do hospital, submetido ao Comitê de Ética em Pesquisa da Universidade Federal de Sergipe (UFS) e aprovado sob CAAE 14521913.3.0000.5546.

\section{RESULTADOS}

Participaram do estudo 22 pacientes, entre 1 e 14 anos, com média de idade de 6,9 anos. Lactentes (menores de 2 anos) representaram 13,6\% da amostra, pré escolares ( 2 anos a 5 anos) $36,4 \%$, escolares (6 anos a 10 anos) $31,8 \%$ e adolescentes (11 anos a 19 anos) 18,2\%. Houve uma predominância do sexo masculino (59\%). 
Foram procedentes da capital 9 pacientes (40,9\%), 11 (50\%) dos demais municípios do interior do estado de Sergipe e dois $(9,1 \%)$ de municípios do interior da Bahia. A maioria residia em zona urbana $(63,6 \%)$ e oito $(36,4 \%)$ foram provenientes da zona rural. Em nove residências $(40,9 \%)$ havia cão em domicílo.

Nenhuma criança tinha diagnóstico anterior de doença crônica, todos tiveram sorologia para o Vírus a Imunodeficiência Humana (HIV) negativa e nenhuma coinfecção foi encontrada.

O tempo médio decorrido entre o início dos sintomas e o internamento foi de 37 dias, sendo o menor tempo entre o aparecimento do primeiro sintoma e o momento da admissão hospitalar de 5 dias, enquanto que 0 máximo foi de 160 dias. Em 45,5\% dos pacientes esse tempo decorrido foi entre 30 e 60 dias.

A frequência de manifestações respiratórias foi de $27,3 \%$. O sintoma respiratório mais observado foi a tosse $(22,7 \%)$, referida como produtiva por quatro pacientes $(18,2 \%)$ e como seca por um $(4,5 \%)$. O segundo sintoma respiratório foi a taquipneia $(9,1 \%)$, que apresentou um caso $(4,5 \%)$ associado à tosse produtiva. $\mathrm{Na}$ população avaliada não foram evidenciados dor torácica ou outros sintomas respiratórios.

As manifestações gerais encontradas, em ordem de frequência, foram: hepatomegalia (100\%), esplenomegalia $(95,5 \%)$, palidez cutânea e de mucosas $(72,7 \%)$, febre $(68,2 \%)$, astenia $(45,5 \%)$, diarreia $(27,3 \%)$ e edema $(4,5 \%)$. Na Tabela 1 estão descritas as manifestações respiratórias e principais manifestações gerais observadas no estudo.

Tabela 1 - Principais manifestações clínicas dos pacientes pediátricos internados com LV, no período de janeiro de 2015 a julho de 2018.

\begin{tabular}{lcc}
\hline Manifestações Clínicas & N (22) & $\%$ \\
\hline Manifestações respiratórias & & \\
\hline Tosse seca & 1 & 4,5 \\
Tosse produtiva & 4 & 18,2 \\
Taquipneia & 2 & 9,1 \\
Dor torácica & 0 & 0 \\
\hline Manifestações gerais & & \\
\hline Hepatomegalia & 22 & 100 \\
Esplenomegalia & 21 & 95,5 \\
Palidez & 16 & 72,7 \\
Febre & 15 & 68,2 \\
Astenia & 10 & 45,5 \\
Diarreia & 6 & 27,3 \\
Edema & 1 & 4,5 \\
\hline
\end{tabular}

Fonte: FRANÇA CM, et al., 2021.

Quanto ao diagnósitco da LV, o teste rápido para Antígeno Recombinante (rK39) foi realizado em todos os pacientes, tendo positividade de $95,5 \%$ (21/22) e o mielograma de $95 \%$ (19/20).

A Tomografia Computadorizada de Alta Resolução (TCAR) foi considerada alterada em $50 \%$ dos casos. As alterações observadas com maior frequência foram opacidades em vidro fosco, opacidades reticulares, linfonodos residuais, opacidades alveolares e derrame pleural. As opacidades em vidro fosco apresentaramse em associação com micronódulos com densidade de partes moles e]m um paciente; com atelectasias e linfonodo residual em um segundo; e com mosaico em um terceiro. As opacidades reticulares apareceram de forma isolada em dois pacientes e opacidades alveolares isolada em um. Um paciente apresentou opacidades alveolares asssociadas com espessamento cisural. O derrame pleural foi visto associado à opacidade em traves e estrias fibroatelectásicas retráteis e linfonodos residuais em um caso e à opacidade reticular em outro. Opacidades micronodulares em árvore em brotamento associadas a espessamento pleural foi encontrado em um paciente. Espessamento do interstício peribroncovascular e um caso de linfonodos residuais foram achados isolados, não associados com outras alterações. Os resultados das TCAR são apresentados na Tabela 2. 
Tabela 2 - Resultados das TCAR dos pacientes pediátricos internados com LV, no período de janeiro de 2015 a julho de 2018.

\begin{tabular}{lcc}
\hline TCAR & N (22) & $\%$ \\
\hline Opacidades em vidro fosco & 3 & 13,6 \\
Opacidades reticulares & 3 & 13,6 \\
Linfonodos residuais & 3 & 13,6 \\
Opacidade alveolares & 2 & 9,1 \\
Derrame Pleural & 2 & 9,1 \\
Atelectasias & 1 & 4,5 \\
Espessamento do interstício peribroncovascular & 1 & 4,5 \\
Espessamento cisural & 1 & 4,5 \\
Espessamento pleural & 1 & 4,5 \\
Micronódulos com densidades de partes moles & 1 & 4,5 \\
Mosaico & 1 & 4,5 \\
Opacidade em traves e estrias fibroelásticas retráteis & 1 & 4,5 \\
Opacidade micronodulares em árvore em brotamento & 1 & 4,5 \\
Sem alterações & 11 & 50 \\
\hline
\end{tabular}

Fonte: FRANÇA CM, et al., 2021.

Não foi encontrada associação entre os sintomas respiratórios e alteração tomográfica, como pode ser observado na Tabela 3. Não foi encontrada associação entre alterações tomográficas e faixa etária (Tabela 4).

Tabela 3 - Associação entre achados tomográficos e sintomas respiratórios em pacientes pediátricos internadas por LV, no período janeiro de 2015 a julho de 2018.

\begin{tabular}{cccc}
\hline Sintomas & \multicolumn{3}{c}{ Tomografia } \\
\cline { 2 - 4 } & $\mathbf{N o r m a l}$ & Alterada & p-valor \\
\hline Tosse seca & $\mathbf{N}(\%)$ & $1(9,1)$ & $1,0000^{\mathrm{F}}$ \\
Tosse produtiva & $0(0)$ & $1(9,1)$ & $0,5865^{\mathrm{F}}$ \\
Taquipneia & $1(9,3)$ & $1(9,1)$ & $1,0000^{\mathrm{F}}$ \\
\hline
\end{tabular}

Legenda: $\mathrm{n}$ - frequência absoluta; \% - frequência relativa percentual; $\mathrm{F}$ Teste Exato de Fisher.

Fonte: FRANÇA CM, et al., 2021.

Tabela 4 - Associação entre faixa etária e alterações tomográficas de pacientes pediátricos internados por LV, no período janeiro de 2015 a julho de 2018.

\begin{tabular}{cccc}
\hline \multirow{2}{*}{ Variaveis } & \multicolumn{3}{c}{ Tomografia } \\
\cline { 2 - 4 } & $\begin{array}{c}\text { Normal } \\
\mathbf{N}(\%)\end{array}$ & $\begin{array}{c}\text { Alterada } \\
\mathbf{N}(\%)\end{array}$ & p-valor \\
\hline Faixa etária & $2(18,2)$ & $1(9,1)$ & $1,0000^{\mathrm{F}}$ \\
\hline <2 Anos & $6(54,6)$ & $2(18,2)$ & $0,1827^{\mathrm{F}}$ \\
2 a 5 Anos & $3(27,3)$ & $4(36,4)$ & $1,0000^{\mathrm{F}}$ \\
6 a 10 Anos & $0(0,0)$ & $4(36,4)$ & $0,0902^{\mathrm{F}}$ \\
\hline
\end{tabular}

Legenda: $\mathrm{n}$ - frequência absoluta; \% - frequência relativa percentual; $\mathrm{F}$ Teste Exato de Fisher.

Fonte: FRANÇA CM, et al., 2021.

Todos os pacientes tiveram radiografia de tórax considerada sem alterações.

O tratamento específico foi realizado com Glucantime ${ }^{\circledR}$ em 13 pacientes $(59,1 \%)$, com anfotericina B em quatro $(18,2 \%)$ e cinco $(22,7 \%)$ iniciaram o tratamento com Glucantime ${ }^{\circledR}$ e terminaram com anfotericina B. O desfecho verificado em todos os casos foi alta médica. O tempo médio de internação foi de 20,18 dias no geral, para aqueles com alteração tomográfica foi de 20,09 dias e sem atleração de 20,27 dias. 


\section{DISCUSSÃO}

A frequência de manifestações respiratórias foi de $27,3 \%$. A tosse $(22,7 \%)$ foi o sintoma respiratório mais observado, referida como produtiva por quatro pacientes (18,2\%) e como seca por um (4,5\%). Nail AM e Imam AM (2013), em uma análise com 71 pacientes LV no Sudão relataram uma frequência de $39.4 \%$ de tosse. Georgiadou SP, et al. (2016) em estudo realizado na Grécia descreveram a tosse como o quinto sintoma mais frequente em pacientes com LV. Queiroz MJA, et al. (2004), em estudo realizado em Recife, encontrou frequência de $42,6 \%$ de tosse. O presente estudo demostrou a tosse como um sintoma frequente em pacientes imunocompetentes com LV, diferente de alguns autores, os quais consideraram que a tosse seria sintoma apenas em pacientes imunossuprimidos (RAINA S E KASHYAP R, 2010; TUON FF, et al., 2009; URBANO-ARANDA Y, et al., 2012).

Cascio A, et al. (2002) descreveram a tosse presente em portares de LV como efeito adverso do uso de antimonial. Nossa investigação detectou a presença sintomas respiratórios antes da instituição do tratamento específico para a parasitose, e, considerando que nenhum paciente relatou doença respiratória pré-existente, a tosse relatada estava associada à infecção pela leishmania.

O segundo sintoma respiratório foi a taquipneia (9,1\%), encontrada em um caso (4,5\%) associada à tosse produtiva, revelando a presença desse sintoma em crianças imunocompetentes com o quadro clássico da LV, diferentemente de estudos anteriores que evidenciaram a taquipneia em apresentações não usuais de casos de LV em imunocompetentes (QUEIROZ MJA, et al., 2004; RAINA S e KASHYAP R , 2010; URBANOARANDA Y, et al., 2012).

No estudo as manifestações clínicas gerais encontradas, em ordem de frequência foram hepatomegalia, esplenomegalia, palidez cutânea e de mucosas, febre, astenia, diarreia e edema. A ordem de frequência diverge em relação a alguns estudos de sintomatologia de LV em crianças, mas os sintomas gerais encontrados são os mesmos já relatados (CASCIO A, et al., 2002; PACE D, et al., 2011; REY LC, et al., 2005; TUON FF, et al., 2009).

As alterações tomográficas observadas com maior frequência foram opacidades em vidro fosco e opacidades reticulares, que são achados associados ao comprometimento pulmonar intesticial, comprovando a existência da pneumonia intesticial já relatada em estudo em necrópsia realizado décadas passadas (DUARTE Ml, et al., 1989). Merece atenção o achado de que esse comprometimento pulmonar pode ser precoce e pode estar implicado em desfechos desfavoravéis, já que o processo inflamatório intersticial em conjunto com uma fraca resposta macrofágica presente na LV pode causar uma das principais complicações da doença, a broncopneumonia (TUON FF, et al., 2009; QUEIROZ MJA, et al., 2004).

As opacidades alveolares, as opacidades nodulares e o derrame pleural encontrados podem ser secundários a infecção bacteriana pulmonar, complicação frequentemente reportada na LV (TOURINHO BD, et al., 2017). Salienta-se que não houve correspondência entre esses achados tomográficos e os achados na radiografia pulmonar, levando-nos a considerar que frequência de pneumonia bacteriana secundária pode ser maior que a encontrada nas radiografias simples.

Não foi encontrada correspondência entre radiografia e TCAR de tórax no que concerne aos achados sugestivos de comprometimento intesticial. Resalta-se que em portadores de LV, os profissionais de saúde devem buscar na radiografia de tórax achados correspondente a infecção bacteriana, complicação frequentemente encontrada, mas principalmente alterações sugestivas de comprometimento pulmonar intersticial, como infiltrados retículos-nodulares periféricos e redução de volume pulmonar (ELICKER B, et al., 2008). Embora a TCAR seja o padrão ouro para a imagenologia pulmonar, a ultrassonografia transtorácica, método diagnóstico menos complexo e menos invasivo (FRANCISCO NETO MJ, et al., 2016), também pode diagnosticar doença pulmonar intestinal e deve ser considerada para avaliação, diagnóstico e seguimento de crianças com LV.

Os achados da presente pesquisa indicam que sintomas respiratórios em crianças podem estar ausentes mesmo diante de comprometimento pulmonar, já que não foi encontrada associação entre os sintomas respiratórios e alterações tomográficas na população pediátrica. Bispo AJB, et al. (2020) demontrou uma 
associação estatisticamente significativa entre tosse seca e TCAR com alterações, considerando a faixa etária adulta e pediátrica. Acreditamos que tal associação deve também acontecer em crianças, mas o amostra relativamente pequena não permitiu comprovar a associação.

O tempo médio de internação foi estaticamente semelhante em pacientes com a TCAR alterada ou normal, reflexo do tempo de internação ser baseado no tratamento estabelecido e critérios de cura (BRASIL, 2017). Tal informação nos leva a crer que o tratamento especifico para a LV leva a recuperação pulmonar, no entanto, estudos de seguimento são necessários para comprovar tal presuposto. A faixa etária dos menores de 5 anos corresponde a $50 \%$ do estudo, refletindo o padrão encontrado no Brasil, em Malta e na Itália (BRASIL, 2017; PACE D, et al., 2011; CASCIO A, et al., 2002). Não foi encontrada associação entre alteração tomográfica e a faixa etária específica, demonstrando que todas as idades estão susceptíveis ao compromentimento pulmonar.

\section{CONCLUSÃO}

Conclui-se que sintomas respiratórios estão presentes e são frequentes $(27,3 \%)$ na LV, assim como como o comprometimento pulmonar (50\%) demonstrado através das alterações tomográficas. Esse estudo destacase por avaliar exclusivamente a população pediátrica na investigação da LV, com enfoque para sintomatologia respiratória e alterações tomográficas, embora não tenha sido encontrado associação entre as duas variáveis. Sempre que possível, deve-se realizar TCAR sem contraste, visto que o comprometimento pode estar presente mesmo na ausência de sintomas e a radiografia convencional de tórax não é capaz de detectar, em sua totalidade, o comprometimento pulmonar existente na LV.

\section{REFERÊNCIAS}

1. BISPO AJB, et al. Pulmonary involvement in human visceral leishmaniasis: Clinical and tomographic evaluation. Online PLoS ONE, 2020; 15(1).

2. BRASIL. Ministério da Saúde. Secretaria de Vigilância em Saúde. Coordenação-Geral de Desenvolvimento da Epidemiologia em Serviços. Guia de Vigilância em Saúde, 2017.

3. BRASIL. Ministério da Saúde. Sistema de Informação de Agravos de Notificação. Datasus. Informações de Saúde. Morbidade e informações epidemiológicas, 2019.

4. BURZA S, et al. Leishmaniasis. The Lancet, 2018; 392(10151): 891-984.

5. CASCIO A, et al. Pediatric visceral leishmaniasis in Western Sicily, Italy: A retrospective analysis of 111 cases. European Journal of Clinical Microbiology and Infectious Diseases, 2002; 21(4): 277-282.

6. CASTRO BC, SILVA JM. Achados Histopatológicos em Pulmão de Hamsters Experimentalmente Infectados com Leishmania (Leishmania) Chagasi. XVI Jornada Acadêmica de Medicina CAMGH-UFMS. Online Anais UFMS, 2009; $1(1)$.

7. DE OLIVEIRA JM, et al. Mortalidade por leishmaniose visceral: Aspectos clínicos e laboratoriais. Revista da Sociedade Brasileira de Medicina Tropical, 2010; 43(2): 188-193.

8. DE QUEIROZ SAMPAIO MJA, et al. Risk factors for death in children with visceral leishmaniasis. PLoS Neglected Tropical Diseases, 2010; 4(11): 3-7.

9. DUARTE MIS, CORBETT CEP. Histopathological and ultrastructural aspects of interstitial pneumonitis of experimental visceral leishmaniasis. Transactions of the Royal Society of Tropical Medicine and Hygiene, 1984; 78(5): 683-688.

10. DUARTE MI, et al. Interstitial pneumonitis in human visceral leishmaniasis. Trans R Soc Trop Med Hyg, 1989; 83(1): 73-76.

11. ELICKER B, et al. High-resolution computed tomography patterns of diffuse interstitial lung disease with clinical and pathological correlation. J Bras Pneumol., 2008; 34(9): 715-744.

12. FRANCISCO NETO MJ, et al. Avanços na ultrassonografia pulmonar. Einstein, 2016; 14(3): 443-448.

13. GEORGIADOU SP, et al. Leishmaniasis revisited: Current aspects on epidemiology, diagnosis and treatment. Journal of Translational Internal Medicine, 2016; 3(2): 43-50.

14. NAIL AM, IMAM AM. Visceral leishmaniasis: Clinical and demographic features in an African population. Pakistan Journal of Medical Sciences, 2013; 29(2): 485-489.

15. PACE D, et al. Manifestations of paediatric Leishmania infantum infections in Malta. Travel Medicine and Infectious Disiease, 2011; 9(1): 37-46.

16. QUEIROZ MJA, et al. Leishmaniose visceral: características clínico-epidemiológicas em crianças de área endêmica Visceral leishmaniasis: clinical and epidemiological features of children in an endemic area ARTIGO ORIGINAL. J Pediatr (Rio J), 2004; 80(2): 141-146. 
17. RAINA S, KASHYAP R. Atypical presentation of visceral leishmaniasis from non-endemic region. Online $\mathrm{J}$ Health Allied Scs, 2010; 9(13).

18. REY LC, et al. Leishmaniose visceral americana (calazar) em crianças hospitalizadas de área endêmica. J. Pediatr. (Rio J.), 2005; 81(1): 73-78.

19. SINGH S, et al. Visceral Leishmaniasis in Children: Diagnosis, Treatment, and Prevention. Journal of Pediatric Infectious Diseases, 2017; 12(4): 214-221.

20. TOURINHO BD, et al. Prognostic factors for death from visceral leishmaniasis in patients treated with liposomal amphotericin B in an endemic state in Brazil. Transactions of the Royal Society of Tropical Medicine and Hygiene, 2017; 111(4): 163-171.

21. TUON FF, et al. In situ immune responses to interstitial pneumonitis in human visceral leishmaniasis. Parasite Immunology, 2009; 31(2): 98-103.

22. URBANO-ARANDA Y, et al. A very unusual cause of persistent cough diagnosed by bronchoscopy. Respiration, 2012; 84(1): 246-2649. 